\title{
FOREWORD
}

\section{Special Section on Networks Software}

Along with the rapid dissemination of the Internet, a lot of new network related services such as peer 2 peer (P2P) applications and social networking service (SNS) have been developed and widely accepted, and enhanced networking functions have been implemented to make networks more reliable and to greatly improve their capacity and performance. These new services and functions are derived by various innovative technologies, among which Networks Software has been playing essential roles.

This is a special section on such Networks Software. Included in this special section are 12 papers that were selected for publication after careful and intensive review of 42 submitted papers. The technical contents of these papers are classified as follows.

(1) Control software for distributed processing system

(2) Service software for mobile and wireless communication

(3) Software technology for P2P applications

These papers are expected to further promote the progress of researches and developments in Networks Software for the coming advanced and fruitful ubiquitous society.

This section has become possible by a lot of efforts by many individuals. As its Guest Editor-in-chief, I would like to express my deep gratitude to the authors of the papers for their research contribution, and I am more than grateful to the reviewers and all the members of the editorial committee for their great efforts related to the review and the acceptance judgment of the papers. Special thanks are due to Messrs. Shoji Kimura and Yoji Yamato for their excellent administrative job for the editorial committee.

Editorial committee members

Secretary: Shoji Kimura (NTT)

Members: Michihiro Aoki (NTT), Kenji Ishida (Hiroshima City Univ.), Yoshiaki Kakuda (Hiroshima City Univ.), Hiroyuki Kasai (NTT DoCoMo), Kei Kato (Oki Electric Industry), Haruko Kawahigashi (Mitsubishi Electric), Yutaka Miyake (KDDI R\&D Labs.), Yoshihiro Niitsu (Shibaura Institute of Technology), Nagao Ogino (KDDI R\&D Labs.), Tadashi Ohta (Soka Univ.), Yoji Yamato (NTT)

Yasushi Wakahara, Editor-in-chief

Yasushi Wakahara (Member) received the B.E., the M.E. and D.E. degrees from The University of Tokyo in 1972, 1974, and 1999, respectively. He joined Kokusai Denshin Denwa Co., Ltd. (KDD) in 1974. Since then he has made researches on facsimile coding system, international digital switching system, network software engineering, intelligent network and communication network designing. He is currently Professor of The University of Tokyo and his current research interests include networking technology and network security. He received the Paper Awards from the Institute of Images Electronics Engineers of Japan and the IEICE in 1982 and 1999, respectively, the Prime Minister Prize of National Invention Awards from Japan Invention and Innovation in 1987 and Maejima Prize from Teishin Kyokai in 1993. $\mathrm{He}$ is a member of the Information Processing Society of Japan, IEEE and AIAA.

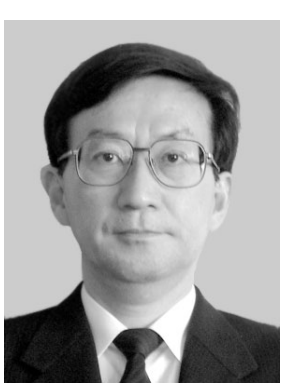

